\title{
Aortenklappenersatz bei älteren Patienten mit schwerer Aortenstenose
}

\author{
Thomas Pilgrim, Peter Wenaweser \\ Klinik und Poliklinik für Kardiologie, Universitätsspital, Inselspital, Bern, Schweiz
}

\begin{abstract}
Summary
Severe calcific aortic stenosis is a disease of the elderly and associated with reduced quality of life and adverse prognosis. According to demographic estimations the share of octogenarians will markedly increase in the course of the decades to come. As a consequence, the incidence of symptomatic aortic stenosis will increase along with the need for minimally invasive treatment options. Since to date there is no efficacious medical treatment for aortic stenosis, elderly patients suffering from significant co-morbidities that portend an increased risk for surgery may benefit from a novel intervention with a minimally-invasive approach. Until this moment, seven years after the first successful transcatheter aortic valve implantation, transfemoral or transapical aortic valves have been implanted worldwide in approximately 10000 patients. Clinical trials and early experience indicate that this minimally invasive intervention proves successful in $>90 \%$. Thirty-day mortality amounts to $10 \%$ and in case of a successful procedure a significant reduction of symptoms and an improvement of quality of life can be expected. Vascular access site complications and atrio-ventricular conduction disturbances are the most commonly observed adverse events.
\end{abstract}

\section{Hintergrund und Demographie der Schweizer Bevölkerung}

Die degenerative Aortenklappenstenose ist die häufigste valvuläre Herzkrankheit und repräsentiert eine klinisch wichtige Herzerkrankung im höheren Alter. Die Bedeutung der Aortenstenose wird in den kommenden Jahrzehnten stark zunehmen, da einerseits bis dato keine wirksame medikamentöse Therapie zur Beeinflussung der Progression der Aortenklappenskle-

Potentieller Interessenkonflikt: PW ist Proctor bei Medtronic CoreValve und Edwards Lifesciences. TP hat keinen Interessenkonflikt im Zusammenhang mit diesem Beitrag. rose zur Verfügung steht und andererseits die Inzidenz der Aortenklappenstenose ab dem 75. Lebensjahr stark ansteigt [1]. Unter dem Gesichtspunkt der sich verändernden Demographie der Schweizer Bevölkerung wird die Aortenklappenstenose im klinischen Alltag

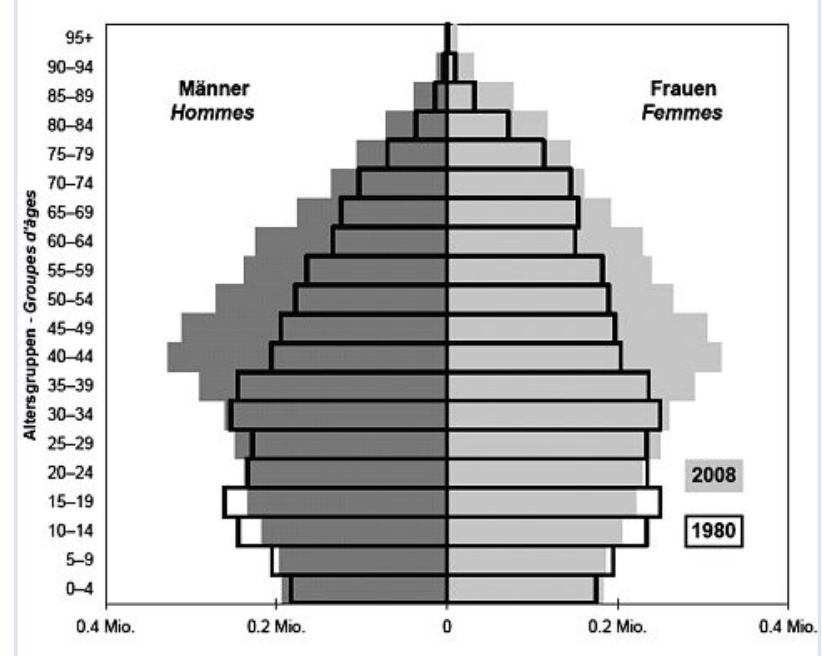

\section{Abbildung 1}

Altersstruktur der Schweizer Bevölkerung. Die Bevölkerungsstruktur der Schweiz hat sich im Verlauf der Jahrzehnte verändert. Die Alterspyramide kann nicht mehr mit einer Tannenform beschrieben werden; stattdessen nimmt sie mehr und mehr die Form einer Art Urne an. Während die Zahl der über 80-jährigen im Jahr 2004328000 betrug und damit einen Anteil von $4 \%$ an der Gesamtbevölkerung darstellte, entwirft das Bundesamt für Statistik für das Jahr 2050 eine Prognose mit 942000 Menschen über 80 Jahren, die 12\% der Gesamtbevölkerung darstellen würden (Quelle: Szenarien zur Bevölkerungsentwicklung der Schweiz, Bundesamt für Statistik).

stark an Bedeutung zunehmen. Erhebungen des Bundesamtes für Statistik zeigen, dass die Alterspyramide (Abb. 1) nicht mehr wie 1980 mit einer Tannenform beschrieben werden kann, sondern mehr die Form einer Urne annimmt. Dies bedeutet, dass die Generation der 40- bis 50-jährigen in der heutigen Schweizer Bevölkerung am Stärksten vertreten ist und die jüngeren Generationen der 10 - bis 20 -jährigen und der 30 - bis 40 -

Korrespondenz:

PD Dr. med. Peter Wenaweser

Leitender Arzt, Invasive Kardiologie

Klinik und Poliklinik für Kardiologie

Universitätsspital, Inselspital

$\mathrm{CH}-3010$ Bern

peter.wenaweser@insel.ch 
Tabelle 1

Schätzungen zur Inzidenz der Aortenstenose.

\begin{tabular}{|c|c|c|c|c|}
\hline & $\begin{array}{l}27 \text { EU-Länder, USA, } \\
\text { Kanada, Japan } \\
2005\end{array}$ & $\begin{array}{l}\text { Schweiz } \\
2005^{c}\end{array}$ & $\begin{array}{l}27 \text { EU-Länder, USA, } \\
\text { Kanada, Japan } \\
2025\end{array}$ & $\begin{array}{l}\text { Schweiz } \\
2036^{d}\end{array}$ \\
\hline Population & 938532000 & 7459128 & 1000108000 & 8200000 \\
\hline Menschen $\geq 65$ Jahre & $15,4 \%$ & $16 \% c$ & $21,6 \%$ & $28 \% d$ \\
\hline Anz. Menschen $\geq 65$ J. $^{a}$ & 144635000 & 1193000 & 217759000 & 2296000 \\
\hline $\begin{array}{l}\text { Inzidenz der Aortenklappensklerose } \\
\text { unter Pat } \geq 65 \mathrm{~J}^{+}\end{array}$ & $26 \%$ & $26 \%$ & $35 \%$ & $35 \%$ \\
\hline Anzahl Pat. mit Aortenklappensklerose & 37605000 & 310000 & 76216000 & 803600 \\
\hline Inzidenz Aortenstenose $(>2,5 \mathrm{~m} / \mathrm{s})^{\mathrm{b}}$ & $2 \%$ & $2 \%$ & $3 \%$ & $3 \%$ \\
\hline Anzahl Pat. mit Aortenstenose & 2893000 & 24000 & 6533000 & 69000 \\
\hline
\end{tabular}

jährigen abgelöst hat. Gleichzeitig beträgt der Anteil der über 80-jährigen aktuell 4\% und derjenige der über 95-jährigen 0,02\% $(n=15800)$ der Gesamtbevölkerung. Szenarien zur Bevölkerungsentwicklung rechnen aufgrund der jährlich zunehmenden Lebenserwartung mit einer massiven Zunahme der über 80-jährigen und prognostizieren einen Anstieg auf 179\% (12\% der Gesamtbevölkerung) bis ins Jahr 2050. Die Lebenserwartung bei Geburt nimmt konstant jährlich zu und betrug 2006 für Frauen 84,0 Jahre und für Männer 79,1 Jahre. Bei Rentenbeginn, mit 65 Jahren wird von einer verbleibenden Lebenserwartung von 21,8 Jahren für Frauen bzw. 19,9 Jahren für Männer ausgegangen [2]. Eindrücklich sind auch die zu erwartenden Kennzahlen für die ganz alten Menschen: Während 1960 in der Schweiz 24 Menschen über 99 Jahre alt waren, werden es im Jahre 2060 voraussichtlich 4303 sein. Erreicht man das stolze Alter von 90 Jahren, beträgt die mittlere Lebenserwartung immer noch 4,3 Jahre für Frauen bzw. 3,9 Jahre für Männer (Stand 1998).

Hochrechnungen aufgrund von Studien zeigen, dass die Prävalenz der symptomatischen Aortenstenose bei über 65 -jährigen bis zu $2 \%$ betragen könnte [3] und diese aufgrund der demographischen Entwicklung auf 3\% im Jahre 2025 ansteigen dürfte (Tab. 1). Auf die Schweizer Bevölkerung bezogen wären dies ca. 24000 Patienten mit einer mittelgradigen bis schweren Aortenstenose im Jahr 2005 und ca. 69000 Patienten im Jahr 2036. Eine schwere Aortenklappenstenose ist nicht nur prognostisch von Bedeutung, sondern bringt auch eine massive Lebensqualitätseinschränkung mit sich, die medikamentös nur wenig beeinflusst werden kann. Der Bedarf einer effektiven, wenn möglich minimal-invasiven Therapie wird deshalb zunehmen.

\section{Pathophysiologie der degenerativen Aortenstenose}

Die degenerative Aortenstenose entwickelt sich über einen Zeitraum von mehreren Jahren bis Jahrzehnten. Genetische Faktoren, Scherkräfte sowie eine nicht trikuspid angelegte Klappenanatomie begünstigen bzw. initiieren die Entwicklung einer Aortenklappensklerose. Läsionen des Klappenendothels erlauben die Einwanderung von T-Zellen, Monozyten sowie LDL in das subendotheliale Gewebe. Angiotensin II sowie unter anderem Interleukin-1 $\beta$ lösen eine phenotypische Transformation von Fibroblasten in Osteoblasten aus, welche zusammen mit dem durch Makrophagen ausgeschütteten Kalzium zu Kalkablagerungen im subendothelialen Gewebe führen [4]. Verschiedene klinische Risikofaktoren wurden mit der Progression der Aortenklappensklerose zu einer schweren Aortenklappenstenose assoziiert. Die wichtigsten sind Alter, männliches Geschlecht, erhöhte Lipidwerte, arterielle Hypertonie, Diabetes mellitus und metabolisches Syndrom sowie Rauchen [3].

\section{Therapeutische Optionen}

Die Behandlungsmöglichkeiten umfassen einerseits medikamentöse, andererseits operative bzw. interventionelle Massnahmen. Vor- und Nachteile der therapeutischen Optionen sind in Tabelle 2 zusammengefasst.

Die medikamentöse Therapie ist bis dato eine rein supportive Massnahme. Verschiedene Studien haben gezeigt, dass beispielsweise die Verabreichung von Statinen [5] oder ACE-Hemmern [6] die Progression der Aortenklappensklerose nicht beeinflussen kann.

Aufgrund der langsamen Progression der Erkrankung, welche eine Anpassung des linken Ventrikels an die sich ändernden Druckverhältnisse erlaubt, wird die 
Tabelle 2

Behandlungsoptionen bei schwerer Aortenklappenstenose.

\begin{tabular}{|c|c|c|}
\hline Behandlungsoption & Pro & Contra \\
\hline $\begin{array}{l}\text { Medikamentös-konservatives } \\
\text { Vorgehen }\end{array}$ & Begrenzte symptomatische Verbesserung & Prognose $\downarrow$ \\
\hline $\begin{array}{l}\text { Chirurgisch-konventioneller } \\
\text { Aortenklappenersatz }\end{array}$ & $\begin{array}{l}\text { Symptomverbesserung } \\
\text { Prognose } \uparrow \\
\text { Langzeitergebnisse }\end{array}$ & $\begin{array}{l}\text { Extrakorporelle Zirkulation } \\
\text { Vollnarkose / Intubation } \\
\text { Sternotomie } \\
\text { Rehabilitationsdauer }\end{array}$ \\
\hline Ballon-Valvuloplastie & Begrenzte symptomatische Verbesserung & $\begin{array}{l}\text { Hohe Restenoserate } \\
\text { Hohe Morbidität }\end{array}$ \\
\hline $\begin{array}{l}\text { Transkatheter Aortenklappen- } \\
\text { Implantation }\end{array}$ & $\begin{array}{l}\text { Symptomverbesserung } \\
\text { Minimal-invasiver Eingriff } \\
\text { Keine Extrakorporelle Zirkulation } \\
\text { Rehabilitationsdauer }\end{array}$ & $\begin{array}{l}\text { z.Z. keine Langzeitdaten } \\
\text { Hohe Schrittmacherbedürfigkeit }\end{array}$ \\
\hline
\end{tabular}

Aortenklappensklerose meist erst im Zustand der schweren Stenosierung symptomatisch und diagnostiziert. Bewirkt der Stenosierungsgrad eine Reduktion der Klappenöffnungsfläche auf $<1 \mathrm{~cm}^{2}$ und zeigt der Patient typische Symptome wie Dyspnoe, Angina pectoris oder Synkopen, ist gemäss den Guidelines die Indikation für einen Aortenklappenersatz gegeben [7]. Bei reduzierter linksventrikulärer Funktion oder inadäquater hämodynamischer Antwort unter Belastung sollte selbst bei fehlenden Symptomen gleichwohl ein Ersatz in Betracht gezogen werden. Als Standardtherapie der schweren, symptomatischen Aortenstenose gilt der operative Aortenklappenersatz mit einer biologischen oder mechanischen Prothese. Erstmals in den 1960er Jahren durchgeführt (Abb. 2), hat sich der operative Aortenklappenersatz als effektive Therapie etabliert und - im Gegensatz zur rein medikamentösen Therapie - die Prognose der Patienten signifikant verbessert [8]. Die Tatsache, dass immer mehr betagte und polymorbide Patienten einer Aortenklappenersatztherapie bedürfen und bei solchen Patienten die klassische Operation mit einer erhöhten Komplikationsrate einhergeht [9, 10], führte zu der Innovation eines interventionellen Aortenklappenersatzes. Vorangehend wurde die Ballonvalvuloplastie als erste interventionelle Massnahme getestet. Diese zeigte jedoch nur einen geringen klinischen Nutzen mit einer mässigen Zunahme der Klappenöffnungsfläche und war assoziiert mit einer beträchtlichen Komplikationsrate [11]. Die meisten Patienten wurden selbst nach prozeduralem Erfolg innert weniger Monate wieder hochsymptomatisch [12].

Im Jahre 2002 gelang die erste perkutane Aortenklappenimplantation nach vorangehender Ballonvalvuloplastie und setzte einen Meilenstein in der Geschichte der kardialen Behandlungsmöglichkeiten [13]. Primär antegrad über einen komplexen, transseptalen Zugang durchgeführt, etablierte sich schon bald der retrograde Zugang über die Arteria femoralis communis [14]. Neben der ballonexpandierenden Edwards-Technologie wurde 2 Jahre später die erste selbstexpandierende Klappenprothese (CoreValve-Revalving System) 


\author{
Abbildung 3 \\ Transapikale Implantation einer Edwards-Sapien-Bioprothese (A-E). \\ A Aortographie. \\ B Vordilatation unter rapid pacing. \\ C Positionierung der auf den Einführungskatheter geladenen Bioprothese. \\ D Klappenimplantation mittels Ballon-Inflation unter rapid pacing. \\ E Kontrollangiographie nach Implantation. \\ 1 Transösophageale Echokardiographie-Sonde; 2 Pigtail-Katheter; 3 Aortenklap- \\ penannulus; 4 transapikale Einführungsschleuse; 5 Edwards-Ballon zur Vordilata- \\ tion der Aortenklappenstenose; 6 Edwards-SAPIEN-Klappe geladen auf Einfüh- \\ rungskatheter; 7 Entfaltete Prothese mit Implantationsballon; 8 Implantierte Aor- \\ tenklappenprothese nach Zurückziehen des Implantationsballones.
}

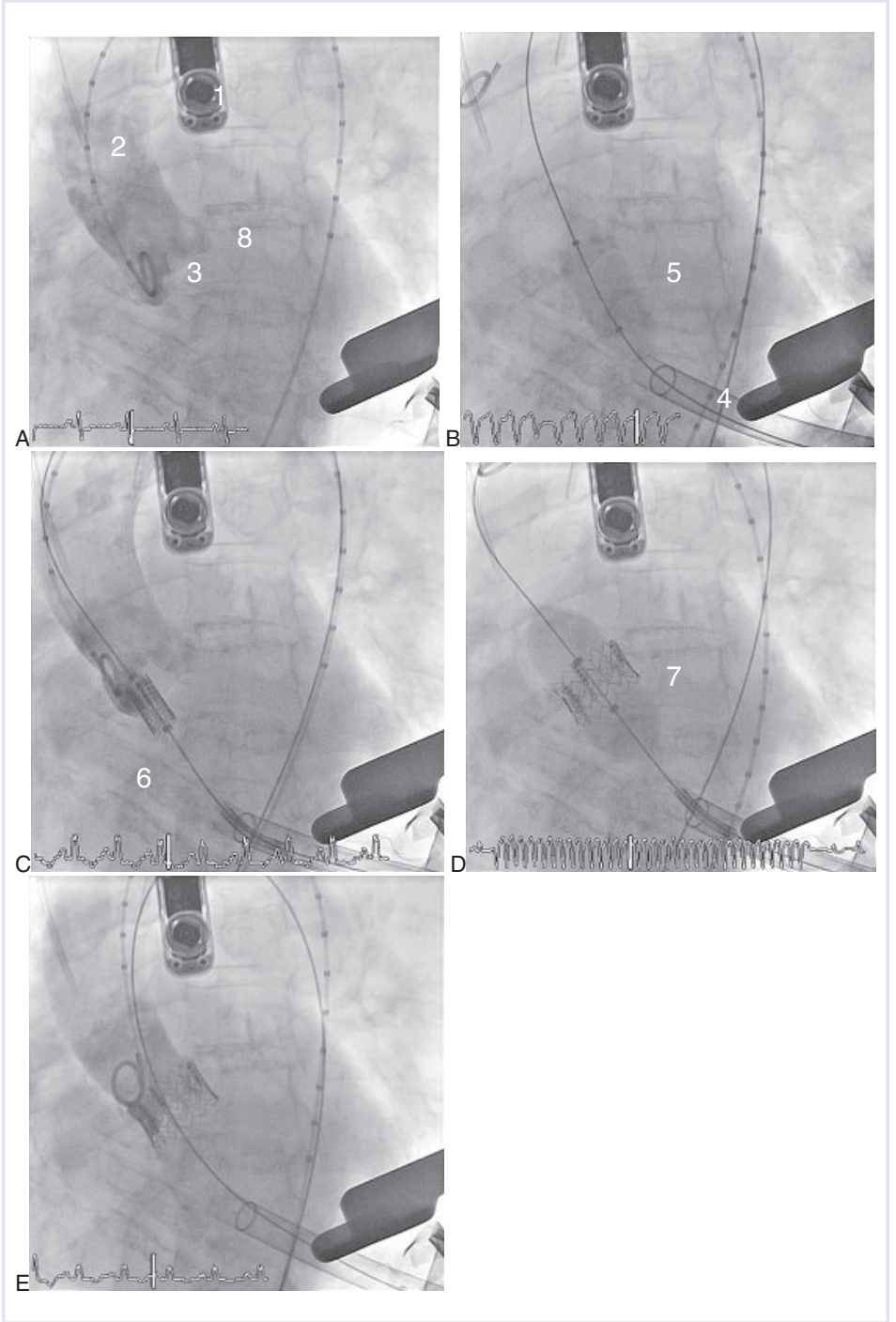

erfolgreich angewandt [15]. Die aktuell noch relativ grosskalibrigen Schleusen zur Einführung der perkutanen Systeme bedingen arterielle ilio-femorale Diameter von mind. 8 bzw. $6 \mathrm{~mm}$. Aufgrund der häufig begleitenden peripheren arteriellen Verschlusskrankheit wurden im weiteren Verlauf alternative Zugänge getestet und auch regelmässig eingesetzt. Der transapikale Zugang für das Edwards-System ist ein etablierter Zugang. Der transaxilläre/transsubklaviale Zugang für des CoreValve-System wurde kürzlich neu eingeführt. Ein Beispiel einer transapikalen und einer transfemoralen Aortenklappenimplantation ist in $\mathrm{Ab}$ bildung 3 und 4 dargestellt. Die wichtigsten unterschiedlichen Merkmale der beiden Systeme sind in Abbildung 5 ersichtlich.

\section{Indikationen für eine Transkatheter- Aortenklappen-Implantation}

Ein perkutaner Aortenklappenersatz, oder besser bezeichnet als TAVI (transcatheter aortic valve implantation), kommt bei Hochrisikopatienten oder für inoperable Patienten in Frage. Das Risiko für einen konventionellen Aortenklappenersatz kann mit Hilfe von verschiedenen Risiko-Scores abgeschätzt werden (Euroscore, STS-Score, Ambler-Score usw.) [16, 17]. Dabei wird primär die berechnete perioperative Mortalität kalkuliert und bei einem Wert über 15-20\% von einer Hochrisikosituation ausgegangen. Diese einfach zu ermittelnden Zahlen bieten jedoch nur eine Hilfestellung und stellen keine definitive Entscheidungsgrundlage im Individualfall dar. So gibt es zum Beispiel verschiedene Risikofaktoren, die für einen konventionell chirurgischen Zugang formal prohibitiv sind, wie beispielsweise eine Vorbestrahlung des Thorax oder eine "Porzellanaorta», welche jedoch in den Risiko-Scores nicht berücksichtigt werden, im Einzelfall jedoch therapieentscheidend sein können. Weitere wichtige Hochrisikofaktoren sind: höheres Alter (>80-jährig), vorgängige Bypass-Operation, relevante Mitralklappeninsuffizienz, schwer reduzierte systolische, linksoder rechtsventrikuläre Funktion, schwere pulmonale Hypertonie, Niereninsuffizienz sowie Demenz bzw. Fragilität des Patienten. Es kann an dieser Stelle nicht genügend darauf hingewiesen werden, dass zum jetzigen Zeitpunkt kein spezifischer Risiko-Score für TAVIPatienten zur Verfügung steht und die Beurteilung der Operabilität oder der Eignung für eine TAVI nicht nur von Risiko-Scores abhängt, sondern einer individuellen Patienteneinschätzung bedarf. Insbesondere wurde gezeigt, dass der häufig angewandte logistische Euroscore die Mortalität überschätzt. Multiple Komorbiditäten bedingen eine multidisziplinäre (Kardiologie, Herzchirurgie, Anästhesie, Hausarzt) Beurteilung des Patienten. Im Falle einer klinischen Indikation für eine TAVI muss zusätzlich die technische Durchführbarkeit überprüft werden. Diese wird anhand von ScreeningUntersuchungen, die hämodynamische, funktionelle und anatomische Faktoren berücksichtigen, evaluiert (Abb. 6, 7).

\section{TAVI: Klinische Resultate}

Die aktuellen klinischen Studienresultate (Tab. 3) zeigen einen technischen Erfolg bei über 90\% der Patienten, wobei die Lernkurve des Operateurs einen Einfluss auf den prozeduralen Erfolg hat. Die 30-TagesMortalität beträgt 8-12\% je nach Patientenpopulation 
und Zugang bei einer geschätzten Mortalität von über 20\% [18-23]. In unserer eigenen Erfahrung mit den beiden Transkatheterklappen, welche 150 Patienten bis Ende November 2009 umfasst, wurde eine erfolgreiche Klappenimplantation bei 98\% der Fälle erreicht. Schwerwiegende Komplikationen wie Tod, Hirnschlag oder Myokardinfarkt traten dabei innert 30 Tagen bei
9\% dieser Hochrisikopatienten auf (geschätzte Mortalität gemäss logistischem EuroSCORE > 20\%). Präliminäre Überlebensanalysen nach TAVI berichten über eine 1-Jahres-Mortalität von ca. 20-30\%. Patienten, welche über den transapikalen Zugang behandelt werden, weisen eine höhere Mortalität auf [24]. Während die periprozedurale Hirnschlagrate bei TAVI-Patienten relativ gering ausfällt $(\sim 2 \%)$, sind vaskuläre Zugangskomplikationen, die bei 4-13\% der Fälle beschrieben worden [25] sind und in engem $\mathrm{Zu}$ sammenhang mit der verwendeten Schleusengrösse stehen, relativ häufig. Zudem wurde gezeigt, dass periprozedurale atrioventrikuläre Überleitungsstörungen eine permanente Schrittmacherimplantation in 5-33\% der Fälle zur Folge haben [24, 26]. Echokardiographische Erhebungen zeigen, dass die immediat guten hämodynamischen Daten im Langzeitverlauf erhalten bleiben und die transvalvulären Gradienten auf einem Niveau von 5-15 mm Hg (mittlerer Gradient) konstant bleiben [24]. Ob eine postprozedurale, häufig beobachtete, leichte bis mässige paravalvuläre Aorteninsuffizienz von klinischer Bedeutung ist, muss im Langzeitverlauf evaluiert werden.

Erfreulich ist, dass gemäss ersten Erhebungen die meisten $\mathrm{Pa}$ tienten schon innert kurzer Zeit weniger symptomatisch sind und die Lebensqualität verbessert wird [27].

Tabelle 3

Veröffentlichte Studien.

\begin{tabular}{|c|c|c|c|c|}
\hline & Webb JG [24] & Grube E [26] & SOURCE-Registry [34] & $\begin{array}{l}\text { Exapanded Evaluation } \\
\text { Registry [20] }\end{array}$ \\
\hline $\begin{array}{l}\text { Klappensystem, } \\
\text { Zugang }\end{array}$ & $\begin{array}{l}\text { Edwards-SAPIEN, } \\
\text { transfemoral/-apikal }\end{array}$ & $\begin{array}{l}\text { CoreValve (18F), } \\
\text { transfemoral }\end{array}$ & $\begin{array}{l}\text { Edwards-SAPIEN, } \\
\text { transfemoral/-apikal }\end{array}$ & $\begin{array}{l}\text { CoreValve (18F), } \\
\text { transfemoral }\end{array}$ \\
\hline Anzahl Patienten & 168 & 102 & 1038 & 646 \\
\hline Alter (Jahre) & $84(79-87)$ & $82 \pm 7$ & $82 \pm 6$ & $81 \pm 7$ \\
\hline Log. EurOSCORE & $29 \%$ & $25 \%$ & $27 \%$ & $23 \%$ \\
\hline Intervention erfolgreich & $94 \%$ & $91 \%$ & $94 \%$ & $97 \%$ \\
\hline \multicolumn{5}{|l|}{ 30-Tages-Outcome } \\
\hline - Mortalität & $11,3 \%$ & $10,8 \%$ & $8,5 \%$ & $8,0 \%$ \\
\hline - Stroke & $4,2 \%$ & $2,9 \%$ & $2,5 \%$ & $1,9 \%$ \\
\hline - Permanenter Schrittmacher & $5,4 \%$ & $33,3 \%$ & $7,0 \%$ & $9,3 \%$ \\
\hline $\begin{array}{l}\text { - Komplikation des vaskulären } \\
\text { Zuganges }\end{array}$ & $6,6 \%$ & nav & $9,3 \%$ & $1,9 \%$ \\
\hline
\end{tabular}




\section{Konventioneller Aortenklappenersatz versus TAVI}

Der konventionelle Aortenklappenersatz hat sich in den letzten Jahrzehnten als standardisierte Therapie mit guten klinischen Ergebnissen etabliert. Während

Abbildung 5

Transkatheter-Aortenklappen-Systeme.

\begin{tabular}{|c|c|c|}
\hline & Edwards-SAPIEN & Medtronic CoreValve \\
\hline \multicolumn{3}{|l|}{ Bioprothese } \\
\hline \multicolumn{3}{|l|}{ Einführungskatheter } \\
\hline Stent-Material & Edelstahl & Nitinol \\
\hline Klappenmaterial & Bovines Perikard & Porcines Perikard \\
\hline Freisetzung & Ballonexpansion & Selbstexpansion \\
\hline Annulusdurchmesser & $18-25 \mathrm{~mm}$ & $19-27 \mathrm{~mm}$ \\
\hline Schleuse & $\begin{array}{l}\text { 22, } 24 \text { French } \\
(18,19 \text { French seit Frühjahr 2010) }\end{array}$ & 18 French \\
\hline Femoralarteriendurchmesser & $28 \mathrm{~mm}$ & $26 \mathrm{~mm}$ \\
\hline Klappengrösse & $23,26 \mathrm{~mm}$ & $26,29 \mathrm{~mm}$ \\
\hline Implantationen weltweit & $>5000$ & $>5000$ \\
\hline Transapikale Implantation & $\mathrm{Ja}$ & Nein \\
\hline Transsubclaviale Implantation & Nein & Ja \\
\hline CE-Zertifizierung & $\mathrm{Ja}$ & Ja \\
\hline
\end{tabular}

Abbildung 6

Vorabklärung für eine Transkatheter-Aortenklappen-Implantation (TAVI)
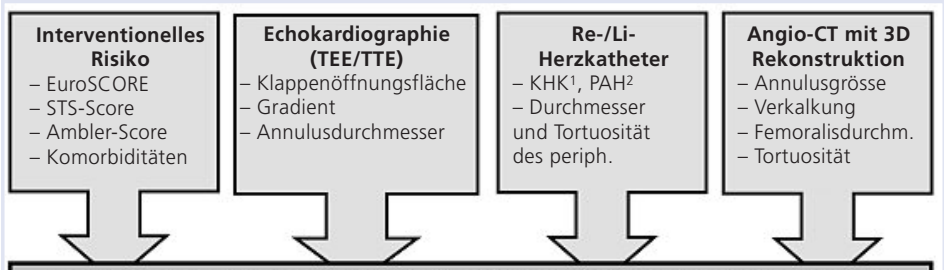

Aufklärungsgespräch, multidisziplinäre Besprechung bezüglich Behandlungsstrategie Transkatheter-Aortenklappen-Implantation

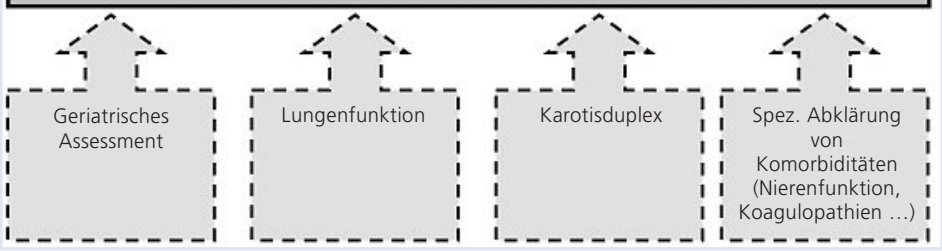

${ }^{1} \mathrm{KHK}=$ koronare Herzkrankheit; $2 \mathrm{PAH}=$ pulmonale Hypertonie.

\section{Abbildung 7}

Vorabklärung vor Transkatheter-Aortenklappen-Implantation (TAVI).

A Angio-CT; Aufsicht auf die verkalkte Aortenklappe.

B Angio-CT mit dreidimensionaler Rekonstruktion des peripheren Zuganges.

C Transösophageale Echokardiographie zur Ausmessung des Aortenannulus.

D Aortograpahie mit Ausmessung des Annulus, des Sinus und des sinotubulären Überganges.
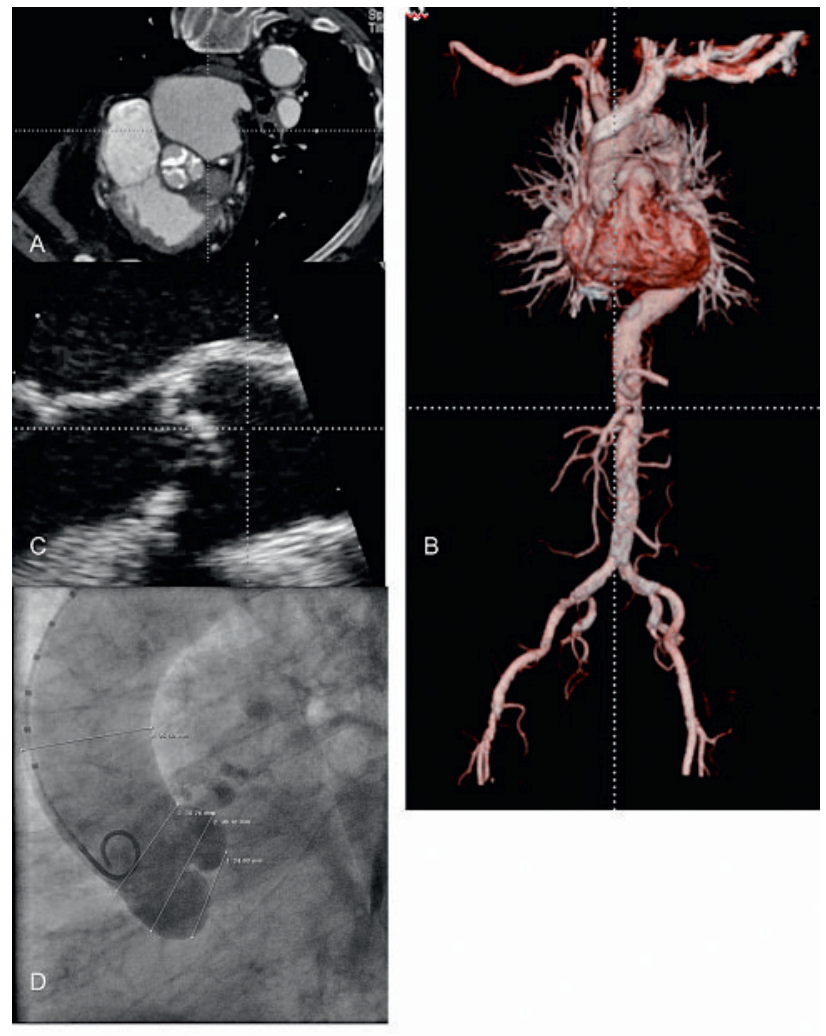

sich das präoperative Mortalitätsrisiko für einen isolierten Aortenklappenersatz in der durchschnittlichen Patientenpopulation auf rund $3 \%$ beläuft [28], erhöhen verschiedene Faktoren wie höheres Alter, schwer eingeschränkte LV-Funktion und vorangehende kardiale Operation (z.b. aorto-koronare Bypass-Operation) das Risiko massgeblich. So berichten retrospektive Analysen von über 80-jährigen Patienten über eine deutlich höhere perioperative Mortalität von 6-12\% [29, 30]. Im Falle einer schwer reduzierten systolischen LV-Funktion steigt das Risiko auf 10-16\% [31, 32]. Die Transkathetertechnologie wurde spezifisch für Hochrisikopatienten entwickelt. Aktuell liegen noch keine Daten von Vergleichsstudien TAVI versus Chirurgie vor. Es sind jedoch Studien im Gange (z.B. US-PARTNERTrial), welche Hochrisikopatienten zwischen TAVI und konventioneller Chirurgie randomisieren. Erste Resultate werden in diesem Jahr erwartet. Ein indirekter Vergleich von konventionell operierten Patienten mit TAVI-Patienten wurde in der sogenannten «Matching study» [33] des Thorax-Zenters in Rotterdam sowie der Universitätsklinik Bern untersucht. Über 1000 konventionell operierte Patienten wurden 100 TAVI-Pa- 
tienten gegenübergestellt. Dabei zeigte sich, dass TAVI-Patienten im Durchschnitt älter, stärker symptomatisch und häufiger voroperiert sind. Zudem war das geschätzte Mortalitätsrisiko bei den TAVI-Patienten deutlich höher. Die 30-Tages-Mortalität für TAVIPatienten betrug knapp 9,8\%, die für die konventionell operierten 2,3\%. Die detaillierte statistische Auswertung ergab, dass die beiden Kohorten aufgrund der ausgeprägt unterschiedlichen Patientencharakteristika nicht direkt miteinander vergleichbar sind und die TAVI-Population bislang vorwiegend medikamentös behandelte Patienten rekrutiert.

Zusammenfassend bietet die neue Transkathetertechnologie einerseits eine minimal-invasive Therapiealternative zur konventionellen Herzchirurgie für Hochrisikopatienten und andererseits eine effektive Behandlung für bis dato nur palliativ, medikamentös therapierte Patienten. Eine gezielte Patientenselektion mittels Vorabklärung in einem erfahrenen Zentrum ist unabdingbar bevor ein solcher Eingriff vorgenommen wird. Die multidisziplinäre Entscheidungsfindung steht dabei im Vordergrund.

\section{Referenzen}

1 Nkomo VT, Gardin JM, Skelton TN, Gottdiener JS, Scott CG, EnriquezSarano M. Burden of valvular heart diseases: a population-based study. Lancet. 2006;368(9540):1005-11.

2 Demographisches Porträt der Schweiz. Ausgabe 2007. Bundesamt für Statistik.

3 Stewart BF, Siscovick D, Lind BK, et al. Clinical factors associated with calcific aortic valve disease. Cardiovascular Health Study. J Am Coll Cardiol. 1997;29(3):630-4.

4 Otto CM. Calcific aortic stenosis - time to look more closely at the valve. N Engl J Med. 2008;359(13):1395-8.

5 Cowell SJ, Newby DE, Prescott RJ, et al. A randomized trial of intensive lipid-lowering therapy in calcific aortic stenosis. N Engl $\mathrm{J}$ Med. 2005;352(23):2389-97.

6 Rosenhek R, Rader F, Loho N, et al. Statins but not angiotensin-converting enzyme inhibitors delay progression of aortic stenosis. Circulation. 2004;110(10):1291-5.

7 Bonow RO, Carabello BA, Chatterjee K, et al. 2008 focused update incorporated into the ACC/AHA 2006 guidelines for the management of patients with valvular heart disease: a report of the American College of Cardiology/American Heart Association Task Force on Practice Guidelines (Writing Committee to revise the 1998 guidelines for the management of patients with valvular heart disease). Endorsed by the Society of Cardiovascular Anesthesiologists, Society for Cardiovascular Angiography and Interventions, and Society of Thoracic Surgeons. J Am Coll Cardiol. 2008;52(13):e1-142.

8 Varadarajan P, Kapoor N, Bansal RC, Pai RG. Clinical profile and natural history of 453 nonsurgically managed patients with severe aortic stenosis. Ann Thorac Surg. 2006;82(6):2111-5.

9 Alexander KP, Anstrom KJ, Muhlbaier LH, et al. Outcomes of cardiac surgery in patients $>$ or $=80$ years: results from the National Cardiovascular Network. J Am Coll Cardiol. 2000;35(3):731-8.

10 Astor BC, Kaczmarek RG, Hefflin B, Daley WR. Mortality after aortic valve replacement: results from a nationally representative database. Ann Thorac Surg. 2000;70(6):1939-45.

11 Percutaneous balloon aortic valvuloplasty. Acute and 30-day follow-up results in 674 patients from the NHLBI Balloon Valvuloplasty Registry. Circulation. 1991;84(6):2383-97.

12 Otto CM, Mickel MC, Kennedy JW, et al. Three-year outcome after balloon aortic valvuloplasty. Insights into prognosis of valvular aortic stenosis. Circulation. 1994;89(2):642-50.

13 Cribier A, Eltchaninoff H, Bash A, et al. Percutaneous transcatheter implantation of an aortic valve prosthesis for calcific aortic stenosis: first human case description. Circulation. 2002;106(24):3006-8.
14 Webb JG, Chandavimol M, Thompson CR, Ricci DR, Carere RG, Munt BI, Buller CE, Pasupati S, Lichtenstein S. Percutaneous aortic valve implnatation retrograde from the femoral artery. Circulation. 2006;113 (6):842-50.

15 Grube E, Laborde JC, Zickmann B, et al. First report on a human percutaneous transluminal implantation of a self-expanding valve prosthesis for interventional treatment of aortic valve stenosis. Catheter Cardiovasc Interv. 2005;66(4):465-9.

16 Kasimir MT, Bialy J, Moidl R, et al. EuroSCORE predicts mid-term outcome after combined valve and coronary bypass surgery. J Heart Valve Dis. 2004;13(3):439-43.

17 Nilsson J, Algotsson L, Hoglund P, Luhrs C, Brandt J. Early mortality in coronary bypass surgery: the EuroSCORE versus The Society of Thoracic Surgeons risk algorithm. Ann Thorac Surg. 2004;77(4):1235-9; discussion 9-40.

18 Grube E, Laborde JC, Gerckens U, et al. Percutaneous implantation of the CoreValve self-expanding valve prosthesis in high-risk patients with aortic valve disease: the Siegburg first-in-man study. Circulation. 2006;114(15):1616-24.

19 Grube E, Schuler G, Buellesfeld L, et al. Percutaneous aortic valve replacement for severe aortic stenosis in high-risk patients using the second- and current third-generation self-expanding CoreValve prosthesis: device success and 30-day clinical outcome. J Am Coll Cardiol. 2007;50(1):69-76.

20 Piazza N, Grube E, Gerckens U, et al. Procedural and 30-day outcomes following transcatheter aortic valve implantation using the third generation (18 Fr) corevalve revalving system: results from the multicentre, expanded evaluation registry 1-year following CE mark approval. EuroIntervention. 2008;4(2):242-9.

21 Walther T, Simon P, Dewey T, et al. Transapical minimally invasive aortic valve implantation: multicenter experience. Circulation. 2007;116(11 Suppl):I240-5.

22 Webb JG, Chandavimol M, Thompson CR, et al. Percutaneous aortic valve implantation retrograde from the femoral artery. Circulation. 2006;113(6):842-50.

23 Webb JG, Pasupati S, Humphries K, et al. Percutaneous transarterial aortic valve replacement in selected high-risk patients with aortic stenosis. Circulation. 2007;116(7):755-63.

24 Webb JG, Altwegg L, Boone RH, et al. Transcatheter aortic valve implantation: impact on clinical and valve-related outcomes. Circulation. 2009;119(23):3009-16.

25 Van Mieghem NM, Nios RJ, Piazza N, et al. Vacular complications with transcatheter aortic valve implantation using the 18 Fr Medtronic CoreValve System: the Rotterdam experience. EuroIntervention. 2010;5: 673-9.

26 Grube E, Buellesfeld L, Mueller R, et al. Progress and Current Status of Percutaneous Aortic Valve Replacement: Results of Three Device Generations of the CoreValve Revalving System. Circ Cardiovasc Intervent. 2008; 1:167-75

27 Ussia GP, Mule M, Barbanti M, et al. Quality of life assessment after percutaneous aortic valve implantation. Eur Heart J. 2009;30(14):1790-6.

28 Society of Thoracic Surgeons national cardiac surgical database. Chicago: Society of Thoracic Surgeons, www.sts.org.

29 Likosky DS, Sorensen MJ, Dacey LJ, et al. Long-term survival of the very elderly undergoing aortic valve surgery. Circulation. 2009;120:S127-33.

30 Leontyev S, Walther T, Borger MA, et al. Aortic valve replacement in octogenarians: utility of risk stratification with EuroSCORE. Ann Thorac Surg. 2009;87:1440-5.

31 Sharony R, Grossi EA, Saunders PC, et al. Aortic valve replacement in patients with impaired ventricular function. Ann Thorac Surg. 2003;75:1808-14.

32 Levy F, Laurent M, Monin JL, et al. Aortic valve replacement for lowflow/low-gradient aortic stenosis operative risk stratification and longterm outcome: a European multicenter study. J Am Coll Cardiol. 2008;51:1466-72.

33 Piazza N, Van Gameren M, Jüni P, et al. A comparison of patient characteristics and 30-day mortality outcomes after transcatheter aortic valve implantation and surgical aortic valve replacement for the treatment of aortic stenosis: a two-centre study. EuroIntervention. 2009;5:580-8.

34 Thomas M, Schmyk G, Walther T, et al. 30 day result of the SOURCE registry: a European registry of transcatheter aortic valve implantation using the Edwards Sapien valve. Presented at the EuroPCR 2009. 